\title{
UNIONS OF HILBERT CUBES
}

BY

\author{
RAYMOND Y. T. WONG(1) AND NE LLY KROONENBERG(2)
}

\begin{abstract}
This paper gives a partial solution to the problem whether the union of two Hilbert cubes is a Hilbert cube if the intersection is a Hilbert cube and a $Z$-set in one of them. Our results imply West's Intermediate Sum Theorem on Hilbert cube factors. Also a technique is developed to obtain $Z$-sets as limits of $Z$-sets.
\end{abstract}

0. Introduction. This paper gives a partial solution to the following problem about the Hilbert cube $Q=[-1,1]^{\infty}$ :

Question 1. Is the union of two copies of the Hilbert cube homeomorphic to the Hilbert cube when their intersection is?

The converse is not the case: let $a$ be an arc in $\{0\} \times Q$ whose complement is not the complement of a point. Then $Q_{1}=([-1,0] \times Q) / \alpha, Q_{2}=$ $([0,1] \times Q) / a$, and $Q_{1} \cup Q_{2}$ are all homeomorphic to $Q$ (since $\alpha$ can be shown to have the complement of a point in $J \times Q$, where $J=[-1,0],[0,1]$ or $[-1,1])$, whereas $Q_{1} \cap Q_{2}$ is not homeomorphic to $Q$.

The analogous problem for Hilbert cube factors is solved by West [8], [9]. $\triangle$ Hilbert cube factor is a topological space $X$ such that $X \times Q \cong Q$. This is equivalent to the existence of a space $Y$ such that $X \times Y \cong Q$. In [8] West proved that the union of two Hilbert cube factors is a Hilbert cubefactor if the intersection is a Hilbert cube factor and a $Z$-set in one of them. (This is the Intermediate Sum Theorem.) A general formal definition of $Z$-set will be given in $\$ 1$, but for the Hilbert cube $Q$, a closed subset is a $Z$-set in $Q$ iff it is equivalent under a space homeomorphism to a subset of an endface. West's result [8] appears here as Theorem 1.4. Observe that from this result it follows immediately that the triod and, more generally, all collapsible polyhedra are Hilbert cube factors.

Using the result of [8], West settles the general question for Hilbert cube

Received by the editors April 19, 1973 and, in revised form, June 6, 1974. AMS (MOS) subject classifications (1970). Primary 54B25, 57A20, 58B05.

Key words and phrases. Union of Hilbert cubes, union of Hilbert cube factors, cap set, fd cap set, inverse limit, near homeomorphism, $G_{\delta}$ in hyperspace.

(1) Supported in part by NSF grant GP 20632.

(2) Supported in part by NSF grant GP $34635 \mathrm{X}$. 
factors proving that any union of two Hilbert cube factors is a Hilbert cube factor if the intersection is [0].

The earliest result for Hilbert cubes is: the union of two copies $Q_{1}$ and $Q_{2}$ of the Hilbert cube is a Hilbert cube if the intersection is a copy of the Hilbert cube and is a $Z$-set in both. This is an easy consequence of the

Homeomorphism Extension Theorem. Any homeomorphism between two $Z$-sets in $Q$ can be extended to an autohomeomorphism of $Q$. Thus we may assume that the intersection is an endface in both, so that the triple $\left(Q_{1}, Q_{2}\right.$, $\left.Q_{1} \cap Q_{2}\right)$ is equivalent to $(Q \times[-1,0], Q \times[0,1], Q \times\{0\})$ under a homeomorphism on $Q_{1} \cup Q_{2}$, and hence $Q_{1} \cup Q_{2} \cong Q$.

In this paper we maintain the condition that $Q_{1} \cap Q_{2}$ is a $Z$-set in $Q_{1}$. However, the requirement that $Q_{1} \cap Q_{2}$ is a $Z$-set in $Q_{2}$ has been relaxed considerably. We use the following notions: a cap set for $Q$ is a subset of $Q$ which is equivalent to the pseudoboundary $B(Q)=\left\{x|\exists i:| x_{i} \mid=1\right\}$. A characterization and examples will be given in $\$ 1$. Furthermore we use $f d$ cap sets, a finite-dimensional analogue of cap set. Both cap sets and $\mathrm{fd}$ cap sets are widely used in infinite-dimensional topology. The main result of $\oint_{1}$ is that the condition that $Q_{1} \cap Q_{2}$ is a $Z$-set in $Q_{2}$ can be replaced by: there is a cap set for $Q_{1} \cap Q_{2}$ which is a countable union of Z-sets in $Q_{2}$ (Proposition 1.3). Also we give a simpler proof of West's first result from [8]. T. A. Chapman independently and earlier discovered the inverse-limit type of proof employed here; he had essentially the same (unpublished) proof for the Intermediate Sum Theorem (here Theorem 1.4). In $\$ 2$ it is shown that, if for a subset $A \subset Q, A \cong Q$, there exists an fd cap set for $A$ which is a countable union of $Z$-sets in $Q$, then this fd cap set can be 'blown up' to a cap set for $A$ which is a countable union of $Z$-sets in $Q$. Therefore the word cap set can be replaced by fd cap set in Proposition 1.3. Combined with a result from [6] this produces Corollary 2.3 to the effect that it is sufficient that $Q_{1} \cap Q_{2}$ has deficiency 1 in $Q_{2}$, or that $Q_{1} \cap Q_{2}$ is nowhere dense in $Q_{2}$ and $Q_{2} \backslash Q_{1}$ is $0-$ ULC and 1-ULC.

1. A preliminary version of the Main Theorem. Proposition 1.3 below is preliminary to Main Theorem 2.2. A closed subset $K$ of a metric space $X$ is a $Z$-set in $X$ if for each $\epsilon>0$ there exists a map $f: X \rightarrow X-K$ such that $d\left(f, \mathrm{id}_{X}\right)<\epsilon$. It is easily seen that any closed subset of a $Z$-set and any finite or countable closed union of Z-sets is again a Z-set. A stronger form of the Homeomorphism Extension Theorem is: Every homeomorphism between two $Z$-sets in $Q$ which moves points less than $\epsilon$ can be extended to an autohomeomorphism on $Q$ which moves points less than $\epsilon$ (see [1], [3]). In the In- 
troduction a cap set of $Q$ was defined as a subset of $Q$ equivalent under an autohomeomorphism of $Q$ to the pseudoboundary $B(Q)=\left\{x|\exists i:| x_{i} \mid=1\right\}$. Now we define (see [2]): A subset $M$ of $Q$ is an (fd) cap set for $Q$ if $M$ can be written as $\bigcup_{i} M_{i}$, where each $M_{i}$ is a (finite-dimensional) Z-set in $Q, M_{i} \subset$ $M_{i+1}$, and such that the following absorption property holds: for all $\epsilon, j$ and every (finite-dimensional) $Z$-set $K$ in $Q$ there is an autohomeomorphism $h$ : $Q \rightarrow Q$ such that $d(h$, id $)<\epsilon,\left.h\right|_{M_{j}}=$ id and $h(K) \subset M_{i}$ for some $i$. Every closed subset of an (fd) cap set is a $Z$-set and every two (fd) cap sets are equivalent under a space homeomorphism. Examples for cap sets are: the pseudoboundary

$$
B(Q), \quad M=\left\{x|\sup | x_{i} \mid<1\right\}=\bigcup_{i}[-1+1 / i, 1-1 / i]^{\infty},
$$

and

$$
P=\left\{x \mid \text { for all but finitely many } i, x_{i} \leq 0\right\}
$$

(it is not obvious from the definition that $P$ and $B(Q)$ are cap sets). Examples for fd cap sets are

$$
\begin{aligned}
s_{f} & =\left\{x \in(-1,1)^{\infty} \mid \text { for all but finitely many } i, x_{i}=0\right\} \\
& =\bigcup_{i}\left\{x\left|\forall_{j} \leq i,\right| x_{j} \mid \leq 1-1 / i \text { and for all } j>i, x_{j}=0\right\}
\end{aligned}
$$

and

$Q_{f}=\left\{x \in Q \mid\right.$ for all but finitely many $\left.i, x_{i}=0\right\}=\bigcup_{i}\left\{x \mid \forall_{j}>i, x_{j}=0\right\}$.

We need two results on inverse limits. An onto map $f: X \rightarrow Y$, where $X$ $\cong Y$ are metric spaces, is a near-homeomorphism if $f$ can be uniformly approximated by homeomorphisms.

Theorem A (Morton Brown [4]). If $x_{1} \stackrel{f_{1}}{\leftarrow} x_{2} \stackrel{f_{2}}{\leftarrow} x_{3} \stackrel{f_{3}}{-} \ldots$ is an inverse sequence, where the $X_{i}$ are homeomorphic compact metric spaces and each $f_{i}$ is a near-homeomorphism, then $\lim _{i}\left(X_{i}, f_{i}\right) \cong X_{1}$.

Lemma B [5] Let $\left(X_{i}, f_{i}\right)$ be an inverse sequence of compact subsets of a compact metric space $X$ and surjections $f_{i}: X_{i+1} \rightarrow X_{i}$, such that foreach $i$

(a) $X_{i} \subset X_{i+1}$ and $\mathrm{Cl}\left(\bigcup_{i} X_{i}\right)=X$,

(b) $d\left(f_{i}, \mathrm{id}_{X_{i+1}}\right) \leq 2^{-i+1}$,

(c) $\left\{f_{i} \circ \ldots \circ f_{j}: X_{j+1} \rightarrow X_{i} \mid j \geq i\right\}$ is an equi-uniformly continuous family of functions (i.e. for each $\epsilon>0$ there is a $\delta>0$ such that for every $j>i$ and every $x$ and $y$ in $X_{j+1}$ with $\left.d(x, y)<\delta, d\left(f_{i} \circ \cdots \circ f_{j}(x), f_{i} \circ \cdots \circ f_{j}(y)\right)<\epsilon\right)$. Then $X \cong \lim _{i}\left(X_{i}, f_{i}\right)$.

Proof. Let $\left(x_{i}\right)_{i} \in \lim \left(X_{i}, f_{i}\right)$ i.e., for each $j, f_{j}\left(x_{j+1}\right)=x_{j}$. Because of (b) there is a map $F: \lim _{i}\left(X_{i}, f_{i}\right) \rightarrow X$ which assigns to $\left(x_{i}\right)_{i}$ its limit. By 
(a) this map is onto and by applying (c) in opposite direction it is 1-1.

If the maps $f_{i}$ in the above lemma do not increase the distance between any two points, then condition (c) of Lemma $B$ is trivially satisfied.

In view of the above, Lemma 1.1 below will be seen to be the core of the proofs of both Proposition 1.3 (the main result of this section) and Theorem 1.4 (West's Intermediate Sum Theorem). The set $Q \times[-1,1]$ will have the metric

$$
d((x, t),(y, s))=|t-s|+\sum_{i} 2^{-i} \cdot\left|x_{i}-y_{i}\right| \cdot
$$

Lemma 1.1. Let $P_{i}=\left\{x \in Q \mid\right.$ for all $\left.j>i, x_{j} \leq 0\right\}$. Let $\tilde{Q}$ be a subset of $Q \times[-1,0]$ which contains, for some given $i, P_{i} \times\{0\}$ as a Z-set. Then $\tilde{Q}$ $\cup\left(P_{i} \times[0,1]\right)$ is homeomorphic to $Q$ and there is a retraction

$$
r_{i-1}: \tilde{Q} \cup\left(P_{i} \times[0,1]\right) \rightarrow \tilde{Q} \cup\left(P_{i-1} \times[0,1]\right)
$$

which is a near-homeomorphism with distance $2^{-i}$ to the identity and does not increase the distance between any two points.

Proof. Since $\tilde{Q} \cap\left(P_{i} \times[0,1]\right)=P_{i} \times\{0\}$ is a $Z$-set both in $\tilde{Q}$ and in $P_{i}$ $\times[0,1]$, we have, by a remark in the Introduction, that $\widetilde{Q} \cup\left(P_{i} \times[0,1]\right)$ is homeomorphic to $Q$. The same is true for $\tilde{Q} \cup\left(P_{i-1} \times[0,1]\right)$.

We define the retraction $r_{i-1}: \widetilde{Q} \cup\left(P_{i} \times[0,1]\right) \rightarrow \widetilde{Q} \cup\left(P_{i-1} \times[0,1]\right)$ as follows:

(1) $r_{i-1} \mid \tilde{Q} \cup\left(P_{i-1} \times[0,1]\right)$ is the identity map;

(2) $r_{i-1}(x, t)=\left(\left(x_{1}, x_{2}, \ldots, x_{i-1}, 0, x_{i+1}, x_{i+2}, \cdots\right), t-2^{-i} \cdot x_{i}\right)$ if $0 \leq 2^{-i} \cdot x_{i} \leq t$

(3) $r_{i-1}(x, t)=\left(\left(x_{1}, x_{2}, \ldots, x_{i-1}, x_{i}-2^{i} \cdot t, x_{i+1}, x_{i+2}, \ldots\right), 0\right)$ if $2^{-i} \cdot x_{i} \geq t$.

In a picture (we show only the $x_{i}{ }^{-}$and $t$-coordinates):

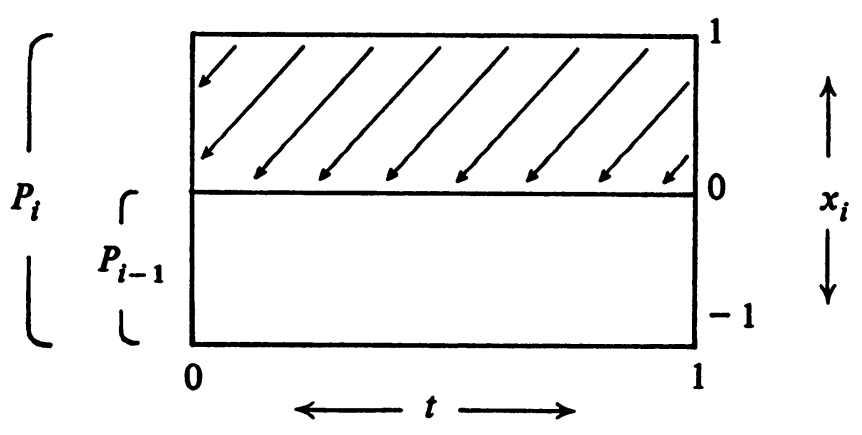

Obviously $d\left(r_{i-1}\right.$, id $)=2^{-i}$. To show that $r_{i-1}$ is a near-homeomorphism we remark that the pair $\left(\widetilde{Q}, P_{i} \times\{0\}\right)$ is homeomorphic to $\left(P_{i} \times[-1,0], P_{i} \times\right.$ 
\{0\}) because of the Homeomorphism Extension Theorem for Z-sets. Thus we get the following picture (again only showing the $x_{i}$-and $t$-coordinates):

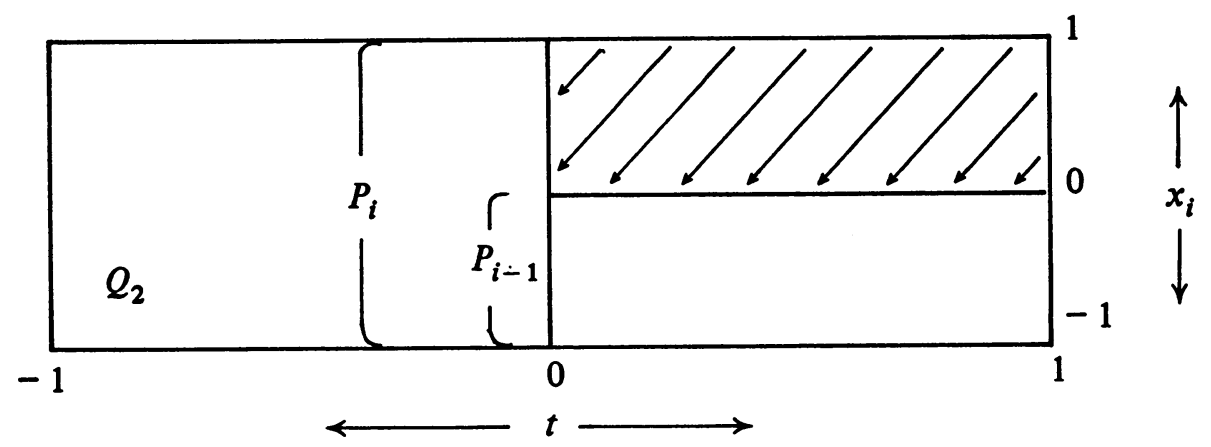

It is easily seen from this picture that $r_{i-1}$ can be approximated by homeomorphisms, also of the form $r_{i-1}^{\prime} \times$ id, where $r_{i-1}^{\prime}$ is defined on the $x_{i}$ and $t$-coordinates and id on the other coordinates.

Finally, it is left as an exercise to the reader that $r_{i}$ does not increase distances.

Lemma 1.2. Let $A \subset Q_{2}$ be homeomorphic to $Q$. Then any homeomorphism $i: A \rightarrow Q \times\{0\}$ can be extended to an embedding of $Q_{2}$ into $Q \times$ $[-1,0]$.

Proof. Let $h: Q_{2} \rightarrow Q \times[-1,0]$ be an arbitrary embedding of $Q_{2}$ onto a $Z$-set. By the Homeomorphism Extension Theorem for $Q \times[-1,0]$ there is a homeomorphism $h^{0}: Q \times[-1,0] \rightarrow Q \times[-1,0]$ which extends $i \circ b^{-1}: h(A)$ $\rightarrow i(A)$. Then $h^{\prime} \circ h$ is an embedding of $Q_{2}$ into $Q \times[-1,0]$ which is an extension of $i$.

We are now ready to prove the preliminary version of the Main Theorem:

Proposition 1.3. If $X$ is $Q_{1} \cup Q_{2}$, where $Q_{1} \cong Q_{2} \cong Q_{1} \cap Q_{2}$ and $Q_{1}$ $\cap Q_{2}$ is a Z-set in $Q_{1}$ such that there is a cap set for $Q_{1} \cap Q_{2}$ which is a countable union of Z-sets in $Q_{2}$, then $X$ is homeomorphic to $Q$.

Proof. We may write $Q_{1}=Q \times[0,1]$, where $Q_{1} \cap Q_{2}$ is identified with $Q \times\{0\}$. By Lemma 1.2, we may also write $Q_{2}=\widetilde{Q} \subset Q \times[-1,0]$. Furthermore we may assume that the cap set postulated in the proposition is the set $P \times$ $\{0\}=\bigcup_{i} P_{i} \times\{0\}$. We form the inverse sequence

$$
\tilde{Q} \cup\left(P_{1} \times[0,1]\right) \stackrel{r_{1}}{\leftarrow} \tilde{Q} \cup\left(P_{2} \times[0,1]\right) \stackrel{r_{2}}{\leftarrow} \tilde{Q} \cup\left(P_{3} \times[0,1]\right) \ldots
$$

with $r_{i}$ as in Lemma 1.1. This inverse sequence satisfies all requirements of Lemma $B$ and therefore shows that $Q_{1} \cup Q_{2}$ is homeomorphic to 
$\lim _{i}\left(\left(\tilde{Q} \cup\left(P_{i} \times[0,1]\right)\right), r_{i}\right)$, whose inverse limit is a Hilbert cube according to Theorem A.

West's Intermediate Sum Theorem [8] can be considered as a corollary to Proposition 1.3, but instead we give a separate proof that bypasses the notion of cap set.

Theorem 1.4 (West [8]). The union of two Hilbert cube factors $X_{1}$ and $X_{2}$, whose intersection is a Hilbert cube factor and a Z-set in $X_{1}$, is a Hilbert cube factor.

Proof. Set $X_{i}^{\prime}=X_{i} \times Q, i=1,2$. Since $X_{1} \cap X_{2}$ is a $Z$-set in $X_{1}$, the definition of Z-set gives immediately that also $X_{1}^{\prime} \cap X_{2}^{\prime}$ is a $Z$-set in $X_{1}^{\prime}$, whereas $X_{1}^{\prime}, X_{2}^{\prime}$, and $X_{1}^{\prime} \cap X_{2}^{\prime}$ are Hilbert cubes. Therefore we can identify $\left(X_{1}^{\prime}, X_{1}^{\prime} \cap X_{2}^{\prime}\right)$ with the pair $(Q \times[0,1], Q \times\{0\})$. Applying Lemma 1.2, we can identify $X_{2}^{\prime}$ with a set $\tilde{Q} \subset Q \times[-1,0]$ which contains $Q \times\{0\}$ and such that $X_{1}^{\prime} \cap X_{2}^{\prime}$ corresponds to $Q \times\{0\}$.

We show that $\left(X_{1}^{\prime} \cup X_{2}^{\prime}\right) \times Q$ is homeomorphic to $Q$ : we form an inverse sequence $\left(Y_{i}, r_{i}^{\prime}\right)_{i}$ where $Y_{i}=(\tilde{Q} \times Q) \cup\left(Q \times[0,1] \times P_{i}\right)$ and $P_{i}$ is defined as in Lemma 1.1. The bonding maps $r_{i}^{\prime}$ are the identity on $\tilde{Q} \times Q$; on the term $Q \times[0,1] \times P_{i}, r_{i-1}^{\prime}$ is identified as $\mathrm{id}_{Q} \times r_{i-1}$, where $r_{i-1}$ is as in Lemma 1.1. Since $P_{i}$ is a $Z$-set in $Q$, also $Q \times\{0\} \times P_{i}$ is a Z-set in $\tilde{Q} \times$ $Q$. Therefore it follows, by a similar argument as in Lemma 1.1 , that $r_{i}^{\prime}$ is a near-homeomorphism and that $Y_{i} \cong Q$. Furthermore, $r_{i}^{\prime}$ does not increase distances. Thus the sequence $\left(Y_{i}, r_{i}^{\prime}\right)_{i}$ satisfies all the conditions of Lemma $B$ and therefore

$$
\left(X_{1} \cup X_{2}\right) \times Q \cong\left(X_{1}^{\prime} \cup X_{2}^{\prime}\right) \times Q \cong \lim \left(Y_{i}, r_{i}^{\prime}\right) \cong Q
$$

Remark. As mentioned in the Introduction, West uses this result (for which he needed a much longer proof) to prove in [ 9 ] the general statement on $Q$-factors.

2. Reduction to fd cap sets. In this section we will describe how to blow up an fd cap set for $Q_{1} \cap Q_{2}$, consisting of $Z$-sets in $Q_{2}$, to a cap set for $Q_{1} \cap Q_{2}$, consisting of $Z$-sets in $Q_{2}$. Before proceeding to this, we need some additional definitions and results:

A set $K \subset Q=[-1,1]^{\infty}$ has deficiency $k$ if $K$ projects onto a point in at least $k$ coordinates; $K$ has infinite deficiency if $K$ projects onto a point in infinitely many coordinates. A subset of $Q$ is topologically equivalent to a closed subset of infinite deficiency iff it is a Z-set in $Q$ [1]. It follows from our definition of $Z$-set (which is closely related to that given in Toruńczyk [7, Theorem 2(a)(ii)], but which is different from Anderson's original definition given in [1]), that the collection of nonempty $Z$-sets forms a $G_{\delta}$ in $2^{Q}$, the hyperspace of $Q$. The proof goes as follows: let $Z_{i}=\left\{K \in 2^{Q} \mid\right.$ 


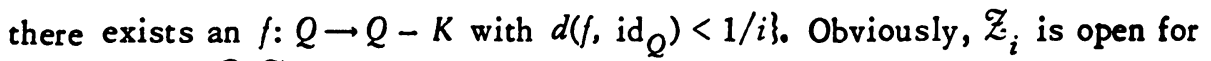
every $i$, and $\bigcap_{i} Z_{i}$ is exactly the collection of all $Z$-sets.

The definition of cap set can be modified somewhat: a countable increasing union $\bigcup_{i} M_{i}$ of $Z$-sets is always a cap set if for every $\epsilon$ there exists a map $h: Q \rightarrow M_{i}$ for some $i$ with $d\left(h, \mathrm{id}_{Q}\right)<\epsilon$ and if moreover for every $i$, $M_{i} \cong Q$ and $M_{i}$ is a $Z$-set in $M_{i+1}$. This can be seen as follows: Since $M_{i}$ $\simeq Q$, every map into $M_{i}$ can be approximated by an embedding onto a $Z$-set in $M_{i}$. So assume that $j \geq 1$ and a $Z$-set $K \subset Q$ are given. Let for some $i>$ $j$ and $h, h: Q \rightarrow M_{i}$ have distance less than $\epsilon / 4$ to the identity. Let $d(g, h)$ $<\epsilon / 4$ and $g$ embeds $Q$ in a $Z$-set in $M_{i}$. Then $d(g$, id $)<\epsilon / 2$. Applying the Homeomorphism Extension Theorem for $M_{i}$, we can extend $\left(g \mid M_{j}\right)^{-1}: g\left(M_{j}\right)$ $\rightarrow M_{j}$ to an autohomeomorphism $f: M_{i} \rightarrow M_{i}$ such that $d(f$, id $)<\epsilon / 2$. Notice that $d(g$, id $)<\epsilon / 2$ iff $d\left(g^{-1}\right.$, id $)<\epsilon / 2$ and also that $d(g$, id $)<\epsilon / 2$ and $d(f$, id $)<$ $\epsilon / 2$ together imply that $d(f \circ g$, id $)<\epsilon$. Thus $f \circ g: Q \rightarrow M_{i}$ has distance less than $\epsilon$ to the identity and leaves $M_{j}$ pointwise fixed. Applying the Homeomorphism Extension Theorem again, but this time to $Q$, we extend $f \circ g \mid K \cup M_{j}$ to the desired homeomorphism $F: Q \rightarrow Q$ with $d(F$, id $)<\epsilon$ and $F \mid M_{j}=$ id.

Lemma 2.1. Let $A \subset Q, A \cong Q$ and suppose $A$ contains an $f d$ cap set $F=\bigcup_{i} F_{i}$ such that each $F_{i}$ is a $Z$-set in $Q$. Then $A$ contains a cap set $M=\bigcup_{i} M_{i}$ such that each $M_{i}$ is a Z-set in $Q$.

Proof. Write $A=[-1,1]^{\infty}$ with metric $d(x, y)=\Sigma_{i} 2^{-i} \cdot\left|x_{i}-y_{i}\right|$ and for each $i, F_{i}=\left\{x \mid\right.$ for all $\left.j>i: x_{j}=0\right\}$. Let $p_{i}$ denote projection onto the $i$ th coordinate and $p_{i}^{\prime}$ projection onto $F_{i}$. We will construct a family $\left\{M_{i}\right\}_{i}$ with for each $i, M_{i} \supset F_{i}, M_{i} \cong Q$ and $M_{i}$ is a $Z$-set in $M_{i+1}$ as well as in $A$ and $Q$. The maps $p_{i}^{\prime}$ and the remarks above show together that $M=\bigcup_{i} M_{i}$ will be a cap set as desired.

First, remark that the collection of nonempty closed subsets of $A$ which are $Z$-sets both in $A$ and in $Q$ form a $G_{\delta}$ in $2^{A}$, too. So write this collection as $Z_{A}=\bigcap_{i} \Theta_{i}$, where each $\Theta_{i}$ is an open subset of $2^{A}$. Since $F_{1}$ is a $Z$-set both in $A$ and in $Q$, there exists an open neighborhood $O_{1}$ of $F_{1}$ such that any closed set $K$ with $F_{1} \subset K \subset O_{1}$ belongs to $\Theta_{1}$. Expand $F_{1}=F_{1}^{(1)}$ to a set $F_{1}^{(2)} \subset F_{3} \cap O_{1}$ of the form $F_{1}^{(2)}=\left\{\left(x_{1}, 0, x_{2}, 0,0, \ldots\right)|| x_{2} \mid \leq c_{2}\right\}$. Choose $\mathrm{O}_{2} \subset \mathrm{Cl}\left(\mathrm{O}_{2}\right) \subset \mathrm{O}_{1}$ such that $\mathrm{O}_{2}$ is an open neighborhood of $F_{1}^{(2)}$ and such that for every closed set $K$ with $F_{1}^{(2)} \subset K \subset O_{2}, K \in \Theta_{2}$. Again choose $F_{1}^{(3)} \subset F_{5} \cap O_{2}$ of the form $\left\{\left(x_{1}, 0, x_{2}, 0, x_{3}, 0,0, \ldots\right\}|| x_{2} \mid \leq c_{2}\right.$ and $\left.\left|x_{3}\right| \leq c_{3}\right\}$. Repeat this construction for all $n$. Let $M_{1}=\operatorname{Cl}\left(\bigcup_{n} F_{1}^{(n)}\right)=$ $\left\{x \mid x_{2 i-1} \leq c_{i}\right.$ and $\left.x_{2 i}=0, i=2,3,4, \cdots\right\}$. Now for all $n, F_{1}^{(n)} \subset M_{1} \subset$ $\mathrm{Cl}\left(O_{n+1}\right) \subset O_{n}$, hence $M_{1} \in \bigcap_{n} \mathcal{O}_{n}$, thus $M_{1}$ is a $Z$-set both in $A$ and in $Q$. Obviously $M_{1} \cong Q$. 
Next we give the construction of $M_{2}$, which is similar to the general inductive step. Since $M_{2}$ will be deficient only in the coordinates divisible by $2^{2}$, we shall be able to prove that $M_{1}$ is a $Z$-set in $M_{2}, M_{1}$ being deficient in all odd coordinates.

Since $F_{2} \cup M_{1}$ is a $Z$-set, there is an open neighborhood $O_{2}^{\prime}$ of $F_{2} \cup$ $M_{1}$ such that for any closed set $K$ with $F_{2} \cup M_{1} \subset K \subset O_{2}^{\prime}, K \in \Theta_{2}$. Expand $F_{2}=F_{2}^{(2)}$ to $F_{2}^{(3)} \subset F_{3} \cap O_{2}^{\prime}$ such that $F_{2}^{(3)} \supset F_{1}^{(2)}=M_{1} \cap F_{3}$, where $F_{2}^{(3)}$ is of the form $F_{2}^{(3)}=\left\{\left(x_{1}, x_{2}, x_{3}, 0,0, \cdots\right)|| x_{3} \mid \leq \phi_{2,3}\left(x_{1}, x_{2}\right)\right\}$ with $\phi_{2,3}$ : $[-1,1] \times[-1,1] \rightarrow(0,1]$ continuous and strictly positive. Choose $O_{3}^{\prime} \subset$ $\mathrm{Cl}\left(\mathrm{O}_{3}^{\prime}\right) \subset \mathrm{O}_{2}^{\prime}$ such that for every closed set $K$ with $F_{2}^{(3)} \cup M_{1} \subset K \subset O_{3}^{\prime}$, $K \in \mathcal{O}_{3}$. Since we want deficiency in all coordinates divisible by 4 , we skip the fourth coordinate and expand $F_{2}^{(3)}$ to $F_{2}^{(4)} \subset F_{5} \cap O_{3}^{\prime}$ such that $\left.F_{2}^{(3)}\right)$ $F_{1}^{(3)}=M_{1} \cap F_{5}$ and $F_{2}^{(4)}$ is of the form $F_{2}^{(4)}=\left\{\left(x_{1}, x_{2}, x_{3}, 0, x_{4}, 0,0, \ldots\right)\right\}$ $\left|x_{3}\right| \leq \phi_{2,3}\left(x_{1}, x_{2}\right)$ and $\left.\left|x_{4}\right| \leq \phi_{2,4}\left(x_{1}, x_{2}, x_{3}\right)\right\}$ with $\phi_{2,4}$ continuous and strictly positive. Going on in this fashion we obtain a family $\left(F_{2}^{(n)}\right)_{n=2}^{\infty}$ and set $M_{2}=\mathrm{Cl}\left(\bigcup_{n} F_{2}^{(n)}\right)$. As before one can show that $M_{2}$ is a Z-set both in $A$ and in $Q$. Using Lemma $B$, it is straightforward to write $M_{2}$ as an inverse limit of finite-dimensional cubes, thus showing that $M_{2}$ is homeomorphic to Q. Also, one can specify a coordinatization for $M_{2}$ such that $M_{1}$ has infinite deficiency in $M_{2}$ and therefore is a $Z$-set in $M_{2}$. The inductive step is completely analogous to the construction of $M_{2}$, with $M_{n}$ containing $F_{n}$ and $M_{n-1}$ and being deficient in all coordinates divisible by $2^{n}$.

It follows from the remarks at the beginning of this section that we have obtained a cap set $M=\bigcup_{n} M_{n}$ for $A$ which is a countable union of $Z$-sets in $Q$.

Main Theorem 2.2. If $X=Q_{1} \cup Q_{2}$, where $Q_{1} \cong Q_{2} \cong Q_{1} \cap Q_{2} \cong Q$; $Q_{1} \cap Q_{2}$ is a $Z$-set in $Q_{1}$ and contains an fd cap set which is a countable union of Z-sets in $Q_{2}$ then $X \cong Q$.

Proof. Combine 1.3 and 2.1.

The potential usefulness of this reduction to $\mathrm{fd}$ cap sets appears in the following corollary (the definition of $i-U \cdot L C$ is given in [6]):

Corollary 2.3. If $X=Q_{1} \cup Q_{2}$ where $Q_{1} \cong Q_{2} \cong Q_{1} \cap Q_{2} \cong Q ; Q_{1} \cap$ $Q_{2}$ is a Z-set in $Q_{1}$ and either has deficiency 1 in $Q_{2}$ or $Q_{2} \backslash Q_{1}$ is $0 . U L C$ and $1-U L C$ and dense in $Q_{2}$, then $X \cong Q$.

Proof. According to 2.4 and 2.5 of [6], in these two cases every closed finite-dimensional subset of $Q_{1} \cap Q_{2}$ is a $Z$-set in $Q_{2}$.

The above corollary is not a very sharp result: we need only one fd cap set for $Q_{1} \cap Q_{2}$ consisting of Z-sets in $Q_{2}$, whereas in the two cases listed every fd cap set for $Q_{1} \cap Q_{2}$ would do. 
Question 2. If $A \subset Q, A \cong Q$, does there exist an fd cap set for $A$ which is a countable union of $Z$-sets in $Q$ ?

Question 2a. If $A \subset Q, A \cong Q$, does $A$ contain a copy of $Q$ which is a $Z$-set in $Q$ ?

From a positive answer to Question 2 it would follow that $Q_{1} \cup Q_{2} \cong$ $Q$ if $Q_{1} \cong Q_{2} \cong Q_{1} \cap Q_{2} \cong Q$ and $Q_{1} \cap Q_{2}$ is a $Z$-set in $Q_{1}$. Another possible line of investigation is trying to relax the conditions on the embedding of $Q_{1} \cap Q_{2}$ in $Q_{1}$. In particular:

Question 3. If $Q_{1} \cong Q_{2} \cong Q_{1} \cap Q_{2} \cong Q$ and $Q_{1} \cap Q_{2}$ contains an fd cap set which is a countable union of Z-sets both in $Q_{1}$ and in $Q_{2}$, then is $Q_{1} \cup Q_{2}$ homeomorphic to $Q$ ?

Question 3a. If $Q_{1} \cong Q_{2} \cong Q_{1} \cap Q_{2} \cong Q$ and $Q_{1} \cap Q_{2}$ contains fd cap sets $M_{1}$ and $M_{2}$ such that $M_{i}$ is a countable union of Z-sets in $Q_{i^{\prime}} i=1$, 2 , then is $Q_{1} \cup Q_{2}$ homeomorphic to $Q$ ?

\section{REFERENCES}

1. R. D. Anderson, On topological infinite deficiency, Michigan Math. J. 14 (1967), 365-383. MR 35 \#4893.

2. - On sigma-compact subsets of infinite-dimensional spaces, Trans. Amer. Math. Soc. (to appear).

3. W. Barit, Small extensions of small homeomorphisms, Notices Amer. Math. Soc. 16 (1969), 295. Abstract \#663-715.

4. M. Brown, Some applications of an approximation theorem for inverse limits, Proc. Amer. Math. Soc. 11 (1960), 478-483. MR 22 \#5959.

5. D. W. Curtis and R. M. Schori, $2^{X}$ and $C(X)$ are homeomorphic to the Hilbert cube, Bull. Amer. Math. Soc. 80 (1974), 927-931.

6. N. Kroonenberg, Characterization of finite-dimensional Z-sets, Proc. Amer. Math. Soc. 43 (1974), 421-427.

7. H. Toruńczyk, Remarks on Anderson's paper: "On topological infinite deficiency", Fund. Math. 66 (1969/70), 393-401. MR 41 \#6149.

8. J. E. West, Infinite products which are Hilbert cubes, Trans. Amer. Math. Soc. 150 (1970), 1-25. MR 42 \#1055.

9. - Sums of Hilbert cube factors (preprint).

DEPARTMENT OF MATHEMATICS, UNIVERSITY OF CALIFORNIA, SANTA BARBARA, CALIFORNIA 93106 (Current address of R. Y. T. Wong)

DEPARTMENT OF MATHEMATICS, LOUISIANA STATE UNIVERSITY, BATON ROUGE, LOUISIANA 70803

Current address (Nelly Kroonenberg): Department of Mathematics, Massachusetts Institute of Technology, Cambridge, Massachusetts 02139 